\title{
Beihilfe zum Suizid oder ärztliche Sterbehilfe?
}

Assisted Suicide or Physician Aid in Dying?

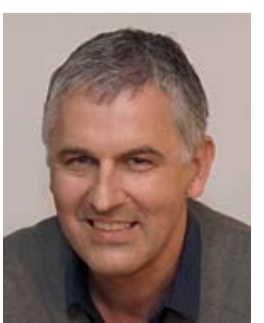

Hermann SpießI

\author{
Autor \\ Hermann SpießI \\ Institut \\ Bezirkskrankenhaus Landshut \\ Bibliografie \\ Psychiat Prax 2020; 47: 411-413 \\ DOI 10.1055/a-1274-1376 \\ ISSN 0303-4259 \\ (c) 2020. Thieme. All rights reserved. \\ Georg Thieme Verlag KG, Rüdigerstraße 14, \\ 70469 Stuttgart, Germany
}

Korrespondenzadresse

Prof. Dr. med. Hermann Spießl, Bezirkskrankenhaus Landshut,

Prof.-Buchner-Straße 22, 84034 Landshut, Deutschland

h.spiessl@bkh-landshut.de
Die Frage, ob und wann es erlaubt sein soll, einem schwer kranken Menschen Hilfe beim Sterben zu geben, stellt sich seit der Antike. Wie Ärzte damit umgehen, hängt von ihrem Berufsverständnis, den Behandlungsmöglichkeiten und den gesellschaftlichen Entwicklungen ab. Seit der Aufklärung wird dem Individuum eine immer größere Selbstbestimmung zugestanden und die Einflussnahme von Staat und Kirche begrenzt.

\section{Das Urteil}

Das Bundesverfassungsgericht (BVerfG) hat mit seinem Urteil vom 26.02.2020 das Verbot der geschäftsmäßigen Förderung der Selbsttötung ( $\$ 217$ StGB) für verfassungswidrig erklärt. §217 StGB greife in das allgemeine Persönlichkeitsrecht ein, das als Ausdruck persönlicher Autonomie auch das Recht auf ein selbstbestimmtes Sterben umfasse. Dies schließe die Freiheit ein, sich das Leben zu nehmen und dabei Angebote von Dritten in Anspruch zu nehmen, und zwar unabhängig von einer bestimmten Schwere einer Erkrankung. Die Entscheidung des Einzelnen, seinem Leben entsprechend seinem Verständnis von Lebensqualität und Sinnhaftigkeit der eigenen Existenz ein Ende zu setzen, sei als Akt autonomer Selbstbestimmung von Staat und Gesellschaft zu respektieren, so die Richter des BVerfG. Der Gesetzgeber müsse die Suizidhilfe so regulieren, dass sichergestellt sei, dass dem Recht des Einzelnen, sein Leben selbstbestimmt zu beenden, hinreichend Raum zur Entfaltung und Umsetzung verbleibt. „Wir mögen seinen Entschluss bedauern, wir dürfen alles versuchen, ihn umzustimmen, wir müs- sen seine freie Entscheidung aber in letzter Konsequenz akzeptieren“, so der Präsident des Bundesverfassungsgerichts Voßkuhle.

\section{Die Stellungnahmen}

Die Reaktionen auf das Urteil des BVerfG waren vielfältig. Die Bundesärztekammer (BÄK) wie auch die Deutsche Gesellschaft für Psychiatrie, Psychotherapie und Nervenheilkunde (DGPPN) betonen, dass die Beihilfe zum Suizid unverändert nicht zu den Aufgaben von Ärzten gehöre und sie nicht verpflichtet werden könnten, an der Selbsttötung mitzuwirken, wie bereits auch das BVerfG ausführte („Niemand kann verpflichtet werden, Suizidhilfe zu leisten“).

Die Aufgabe von Ärzten sei es, unter Achtung des Selbstbestimmungsrechts des Patienten Leben zu erhalten, Gesundheit zu schützen und wiederherzustellen sowie Leiden zu lindern und Sterbenden bis zu ihrem Tod beizustehen, so der Präsident der BÄK. Die BÄK ist damit in voller Übereinstimmung mit der Deklaration der World Medical Association (WMA) von 2019 (gegen aktive Sterbehilfe und assistierten Suizid) und steht ganz in der ärztlichen Tradition von Hufeland und des hippokratischen Eides („Ich werde niemandem, auch nicht auf seine Bitte hin, ein tödliches Gift verabreichen oder auch nur dazu raten."), wie sie sich auch in der aktuellen (Muster-)Berufsordnung für Ärzte (MBO-Ä) widerspiegelt („Ärztinnen und Ärzte haben Sterbenden unter Wahrung ihrer Würde und unter Achtung ihres Willens beizustehen. Es ist ihnen verboten, Patientinnen und Patienten 
auf deren Verlangen zu töten. Sie dürfen keine Hilfe zur Selbsttötung leisten“). Angemerkt sei, dass auch die MBO-Ä entsprechend dem BVerfG-Urteil wohl angepasst werden muss.

Die Deutsche Gesellschaft für Psychiatrie, Psychotherapie und Nervenheilkunde (DGPPN) fokussiert in ihrer Pressemitteilung vom 27.02.2020 auch auf eine zukünftig neue Rolle des Psychiaters als Gutachter, der potenziell entscheiden müsse, ob der Sterbewillige im Hinblick auf seinen Sterbewunsch einen freien, uneingeschränkten Willen habe und zu einer Selbstbestimmung fähig sei. Der Psychiater entscheide somit über Leben und Tod und liefe Gefahr instrumentalisiert zu werden. Da $90 \%$ der Suizide vor dem Hintergrund von psychischen Erkrankungen geschehen (Anmerkung: Diese „Tatsache“ ist durchaus diskutabel [1, 2]), müsse der Schwerpunkt anstelle der Sterbehilfe auf Suizidprävention und auf den „Kampf um das Leben jedes einzelnen Menschen“ gelegt werden. Man könnte meinen, C. W. Hufeland spräche nach über 200 Jahren weiterhin zu uns, der Arzt „dürfe nichts anders thun als Leben erhalten; ob es ein Glück oder Unglück sey, ob es Werth habe oder nicht..."[3].

Für das Nationale Suizidpräventionsprogramm für Deutschland (NaSPro), die Deutsche Akademie für Suizidprävention (DASP) und die Deutsche Gesellschaft für Suizidprävention (DGS) ergibt sich - unter Berücksichtigung der persönlichen Autonomie, aber auch der in Krisen bestehenden Ambivalenz aus dem Urteil die Notwendigkeit, die Suizidprävention zu stärken (Pressemitteilung vom 26.02.2020). Suizidprävention unterstütze Menschen bei selbstbestimmten Entscheidungen über ihr Leben durch respektvolles Verstehen, Beraten und Behandeln, dies schließe auch Angehörige mit ein. Das BVerfG habe die Selbstbestimmung betont, nicht aber deren Einschränkung durch psychische und körperliche Krankheiten und massive Krisen ausreichend gewürdigt und den Schutz von Menschen, die in diesen Lebenssituationen in ihrer Urteils- und Entscheidungsfähigkeit stark eingeschränkt seien, nicht befördert. Menschen in Krisen und bei psychischer Erkrankung bräuchten Hilfen, wie sie auch am Ende des Lebens durch Palliativmedizin, Hospizarbeit, Beratung und Psychotherapie gegeben seien.

Der Vollständigkeit halber sei erwähnt, dass das BVerfGUrteil zum §217 StGB auch seitens der christlichen Kirchen, des Deutschen Ethikrates und der Palliativmedizin kritisch kommentiert wurde. Möglicherweise spielt in Deutschland im Hintergrund weiterhin eine historische Befangenheit aufgrund der im NS-Regime praktizierten „Euthanasie“ eine Rolle, wobei diese Krankenmorde keinesfalls mit aktiver Sterbehilfe oder assistiertem Suizid gleichzusetzen sind.

\section{Ein anderer Blickwinkel}

Begriffe wie Förderung der Selbsttötung, Hilfe zur Selbsttötung, Beihilfe zum Suizid bzw. assistierter Suizid, Tötung auf Verlangen oder Euthanasie werden in der breiten Diskussion teils synonym verwendet, Suizid und Sterbehilfe oft (bewusst?) in einen Topf geworfen. Ist das richtig? Oder versperrt es uns möglicherweise einen „differenzialdiagnostischen“ Blick auf 2 unterschiedliche Problembereiche und damit auf eine Lösung des Dilemmas, das nun gerade auch für die Psychiatrie und die Suizidprävention entstanden ist?

Die American Association of Suicidology (AAS) hat 2017 in ihrem Statement, 15 Unterschiede zwischen Suizid und Ärztlicher Sterbehilfe (Physician Aid in Dying - PAD) herausgearbeitet [4]. Wesentliche Merkmale von Patienten, die um Sterbehilfe bitten, im Gegensatz zu suizidalen Patienten sind m. E. folgende:

- Sie leiden an einer terminalen körperlichen Erkrankung, ihre Lebenserwartung beträgt - entsprechend aktuellem medizinischen Wissenstand - nur noch wenige Monate.

- Es sind keine Patienten mit primär seelischen Erkrankungen, sie zeigen auch keine der üblichen Risikofaktoren für einen Suizid.

- Die Patienten möchten an sich leben, kämpften oft verzweifelt darum; der Verlauf der körperlichen Erkrankung führt aber unweigerlich zum Tod.

- Diese Patienten wissen um alle Möglichkeiten anderer Hilfen und können diese Alternativen vernünftig beurteilen und ihre Entscheidung treffen.

- Sie sind in der Lage, Pro und Kontra ihres vorzeitigen Sterbens vernünftig und objektiv nachvollziehbar abzuwägen; dieser Entscheidungsprozess erfolgt über einen längeren Zeitraum.

- Sie erhalten sich bis zuletzt ihre persönliche Würde und leben bzw. sterben entsprechend ihrer Wertvorstellungen.

Diese Patienten sterben nicht allein, sondern meist zu Hause im Kreise ihrer Familie. Sie müssen dazu nicht harte Methoden wie Erhängen oder Sprung aus großer Höhe anwenden und vermeiden so einen schmerzhaften Tod. Sie erhalten bis zuletzt viel emotionalen Zuspruch von ihren Angehörigen. Letztere sind durch einen solchen Tod auch deutlich weniger belastet oder gar traumatisiert als Angehörige von Suizidenten. Ein solches Sterben ist auch bei weitem nicht so (negativ) öffentlichkeitswirksam und stigmatisiert wie ein Suizid.

Etwas zu vereinfacht erscheint die Darstellung der AAS im Hinblick auf psychische Erkrankungen, hier ist die Abgrenzung zwischen von Suizid und Sterbehilfe deutlich schwieriger [5]. Einer ärztlichen Sterbehilfe bei ausschließlich psychischer Erkrankung - sie macht in den Niederlanden und Belgien ca. 1-2\% der ärztlichen Sterbehilfe aus - müssten aus meiner Sicht grundsätzlich dieselben Voraussetzungen wie bei einer terminalen körperlichen Erkrankung zugrunde gelegt werden, neben der freien Willensentscheidung, insbesondere unerträgliches Leid, fehlende Therapierbarkeit und infauste Prognose. Die medizinethische Diskussion zur ,Psychiatric PAD“ ist gerade im Gange [6-9], insbesondere auch im Fall einer im Voraus verfügten Willenserklärung bei später eintretender Demenz [10, 11].

Trotz möglicher Überschneidungen sollte versucht werden, diese unterschiedlichen Phänomene voneinander abzugrenzen - inhaltlich, aber auch bezüglich ihrer Häufigkeit (d.h. diese Fälle sollten in den Statistiken nicht bei den Suiziden, sondern separat bzw. bei ihren Grunderkrankungen erfasst werden) sowie natürlich in der klinischen Praxis des Arztes und auch des Psychiaters. Sowohl der Ausschluss (behandelbarer) seelischer Erkrankungen und die (Differenzial)Diagnostik der Suizidalität 
wie auch die Feststellung des freien Willens, in Summe die Feststellung der Freiverantwortlichkeit der Entscheidung [12], sind am besten durch einen Psychiater möglich. Wer sonst könnte diese verantwortungsvolle Aufgabe besser bewerkstelligen? Oder wem sollte man sie denn überlassen, wenn man einen Arzt nur dem Leben verpflichtet sieht?

\section{Auswirkungen auf die Suizidprävention}

Die Akzeptanz, dem Sterbewunsch eines Kranken mit freiem Willen zu folgen, hat natürlich Auswirkungen auf die Suizidprävention [6, 13]. Diese darf m. E. aber keine absolute sein, ein gänzlicher Rückzug bei terminal körperlich Kranken, wie es die AAS sieht [4], ist allerdings nicht der beste Weg. Fürsorge für den Patienten und Respekt vor seiner Autonomie sollten sich ergänzen. Natürlich soll einem an einem Karzinom erkrankten alten Menschen grundsätzlich dieselbe Hilfe der Suizidprävention zur Verfügung stehen wie einem jungen Mädchen nach Trennung vom Freund oder einem hoffnungslosen, schwer depressiven 50-Jährigen. Bei letzteren sind alle Maßnahmen, nach meiner (paternalistischen) Meinung auch ggf. gegen deren (nicht mehr freien) Willen, einzusetzen, da hier eine Freiverantwortlichkeit der Suizidentscheidung nicht besteht; dem Sterbewunsch darf hier nicht gefolgt werden. Bei ersterem muss nach dem Angebot und dem Ablehnen der Hilfen sein freier, nicht von psychischer Erkrankung oder Dritten beeinflusster, fester und dauerhafter Wille akzeptiert werden. Das ist ein Akt von Menschlichkeit und erfolgt am besten ärztlich begleitet. Und es ist kein Versagen der Suizidprävention.

Speijer und Diekstra haben bereits in den 70er-Jahren Kriterien erarbeitet, anhand derer entschieden werden könnte, wann ein Suizid nicht unbedingt verhütet werden sollte [14]:

- Die Wahl, das Leben durch Suizid zu beenden, basiert auf einer freien Willensentscheidung des betreffenden Menschen und geschieht nicht unter Druck von anderen.

- Der Zustand dieses Menschen kann als Zustand unerträglicher körperlicher und/oder seelischer Schmerzen beschrieben werden und eine Besserung ist vernünftigerweise nicht zu erwarten.

- Der Wunsch zu sterben kann als unerschütterlich einwandfrei festgestellt werden.

- Dieser Mensch ist zu der Zeit, wo er sich entscheidet Suizid zu begehen, compos mentis, d. h. im vollen Besitz seiner geistigen Kräfte.

- Der Suizid sollte in einer Weise durchgeführt werden, die anderen keinen unnötigen und verhinderbaren Schaden zufügt.

- Der/die Helfende sollte im Gesundheitswesen qualifiziert sein. Sollte eine tödliche Dosis eines Medikaments verschrieben werden müssen, müsste immer ein Arzt beteiligt sein.

- Schließlich sollte jeder Schritt, der getan wird, voll dokumentiert sein und die Dokumente sollen für die zuständigen Behörden bereitgestellt werden.

Prof. Dr. Christian Scharfetter, sein Buch „Allgemeine Psychopathologie“ begleitet(e) viele von uns durch unser Psychiater-
Leben, erkrankte Jahre nach seiner Emeritierung an einem nicht behandelbaren Gallengangskarzinom, die Prognose war infaust und er verstarb 2013. In der für ihn posthum veranstalteten Gedenkfeier berichtete sein Zwillingsbruder, ein Neurochirurg, über seinen Leidensweg und seinen Entschluss, sich durch ihn begleitet das Leben zu nehmen [15]. Vielmehr als jede medizinische, ethische oder religiöse Diskussion zeigt uns dieser Bericht, was Sterben in Würde ohne unerträgliche und unendlich erscheinende Qual bedeutet. Es ist eine Abkürzung des unvermeidbaren Sterbeprozesses. Das kann ärztliche Sterbehilfe leisten und das ist kein Suizid.

Interessenkonflikt

Der Autor gibt an, dass kein Interessenkonflikt besteht.

Literatur

[1] Wolfersdorf M. Sind Menschen, die sich das Leben nehmen, psychisch krank? - Pro. Psychiat Prax 2020; 47: 176-177

[2] Brieger P, Menzel S. Sind Menschen, die sich das Leben nehmen, psychisch krank? - Kontra. Psychiat Prax 2020; 47: 177-178

[3] Hufeland CW. Die Verhältnisse des Arztes. Neues Journal der practischen Arzneikunde und Wundarzneiwissenschaft 1806; 23: 5-36

[4] American Association of Suicidology. "Suicide" is not the same as "physician aid in dying". http://www.suicidology.org/Portals/14/ docs/Press\%20Release/AAS\%20PAD\%20Statement\%20Approved\% 2010.30.17\%20ed\%2010-30-17.pdf

[5] Kim SYH, Conwell Y, Caine ED. Suicide and physician-assisted death for persons with psychiatric disorders how much overlap? JAMA Psychiatry 2018; 75: 1099-1100

[6] Kious BM, Battin MP. Physician aid-in-dying and suicide prevention in psychiatry: a moral crisis? Am J Bioeth 2019; 19: 29-39

[7] Van de Vathorst S. Concerning the basic idea that the wish to end suffering legitimates physician aid in dying for psychiatric patients. Am J Bioeth 2019; 19: 1-2

[8] Nicolini ME, Gastmans C, Kim SYH. Parity arguments for 'Physician Aid-in-Dying' (PAD) for psychiatric disorders: their structure and limits. Am J Bioeth 2019; 19: 3-7

[9] Schneeberger AR, Hoff P, Seifritz E et al. Deciding to end one's life because of a psychiatric illness - a decision without second thoughts? Front Psychiatry 2020; 11: 58

[10] Widdershoven G. Aktive Lebensbeendigung bei einer Patientin mit Alzheimer-Demenz in den Niederlanden: eine ethische Debatte - Pro. Psychiat Prax 2019; 46: 428-430

[11] Steinert T. Aktive Lebensbeendigung bei einer Patientin mit Alzheimer-Demenz in den Niederlanden: eine ethische Debatte - Kontra. Psychiat Prax 2019; 46: 430-431

[12] Cording C, Saß H. Die Freiverantwortlichkeit der Entscheidung für einen assistierten Suizid. NJW 2020; 73: 2695-2697

[13] Mishara BL, Weisstub DN. Is suicide prevention an absolute? Considerations when medical assistance in dying is an option. Crisis 2018; 39 : $313-317$

[14] Diekstra RF. The significance of Nico Speijer's suicide: How and when should suicide be prevented? Suicide Life-Threat Behav 1986; 16: 1315

[15] Scharfetter F. Persönliche Worte zu Krankheit und Sterben von Christian Scharfetter. SANP 2014; 165: 64-68 